\title{
MHD simulations of ram pressure stripping of disk galaxies
}

\author{
Mariana Ramos-Martínez and Gilberto C. Gómez \\ Instituto de Radioastronomía y Astrofísica, UNAM, Apartado Postal 3-72, 58089 Morelia, \\ México \\ email: m.ramos@crya.unam.mx
}

\begin{abstract}
The removal of ISM of disk galaxies through ram pressure stripping (RPS) has been extensively studied in numerous simulations (see Roediger 2009 and references therein). The models show that RPS has a significant impact on galaxy evolution (truncation of the ISM will lead to a decrease in star formation and a change in galaxy color). Nevertheless, the role of magnetic fields (MFs) on the dynamics of the gas in this process has not been sufficiently studied, although the influence of the MFs on the large scale structure is well established. This motivated us to perform a 3D MHD simulation of a disk galaxy with an isothermal, non-self gravitating and magnetized gaseous disk in equilibrium with a galaxy potential (Allen \& Santillán, 1991). We model RPS on the galactic disk under the wind-tunnel approximation with the use of the RAMSES code (Teyssier, 2002) in order to understand the effects of MFs in RPS.
\end{abstract}

Keywords. galaxies: evolution, intergalactic medium, galaxies: magnetic fields.
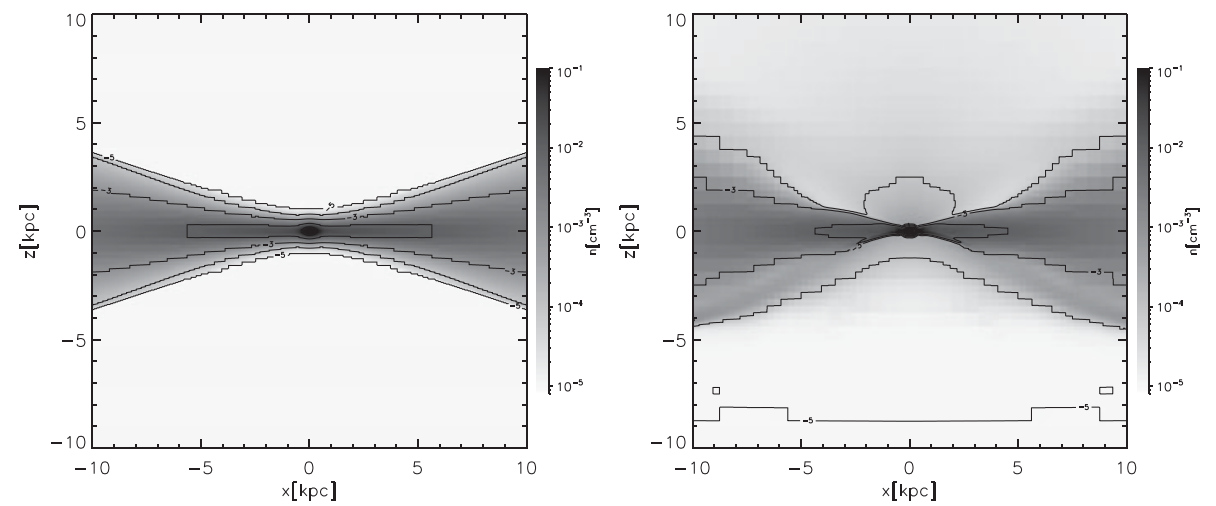

Figure 1. Gas density cut (at $y=0$ ) in log-scale, with isodensity contours, for $t=0$ Myr (left) and $t=134 \mathrm{Myr}$ (right). In the initial condition, the density in the center of the disk decays faster in the $z$ direction than in the outer parts, so our disk presents a flaring due to the galactic magnetic field. The intracluster medium (ICM) wind flows up from bottom of the box, and interacts with the magnetized gas of the galaxy, producing oblique shocks below the mid-plane, which lead to an inflow of gas towards the galactic center. Additionally, an enhancement of the MF intensity in the shocked gas is observed in the simulations.

\section{References}

Allen, C. \& Santillán, A. 1991, Rev. Mexicana AyA, 22, 255

Gómez, G. C. \& Cox, D. 2002, ApJ, 580, 235

Roediger, E. 2009, $A N, 330,888$

Teyssier, R., 2002, AËA, 385, 337 